\title{
Color Doppler Evaluation of Cerebral - Umbilical Pulsatility Ratio and its Usefulness in the Diagnosis of Intrauterine Growth Retardation and Prediction of Adverse Perinatal Outcome
}

\author{
S MAHMOOD ${ }^{\mathrm{a}}$, S CHOWDHURY ${ }^{\mathrm{b}}, \mathrm{SB}^{\mathrm{C}}$ CHOWDHURYc
}

\begin{abstract}
Summary:
The purpose of this study is Evaluation of cerebral umbilical pulsatility ratio by color Doppler and to estimate the value of pulsatility index ratio of cerebral umbilical vessels in the diagnosis of small-for-gestational-age (SGA) fetuses and in the prediction of adverse perinatal outcome. Validity of the test were confirmed by determining sensitivity, specificity, accuracy, positive predictive value, negative predictive value. The study population comprised 40 pregnancies of 30-41 weeks gestation that had been diagnosed clinically as intrauterine growth retardation (IUGR) over a period of 1 year. The cerebral - umbilical pulsatility ratio (C/U ratio) were calculated. The pregnancies were followed up and the final perinatal outcome of each case were noted. Various intraparum and neonatal indicators were used to assess the outcome, with an adverse outcome being defined as the
\end{abstract}

Introduction:

Intrauterine growth retardation is characterized by failure of the fetus to reach its normal growth potential. Intrauterine growth retardation is the second leading cause of perinatal death (Wolfe et al. 1989) ${ }^{1}$. IUGR is associated with significant morbidity including increased rates of meconium aspiration, hypoglycaemia, respiratory distress syndrome, intrapartum asphyxia, developmental delay and still birth (Berkowitz et al. 1988)2 .

Intrauterine growth retardation is associated with an increased risk of perinatal mortality and morbidity and impaired neurodevelopment (Kok et al. 1996) ${ }^{3}$. The correct detection of the compromised Intrauterine

a. Dr. Sharmeen Mahmood, Associate Professor, Department of Obst. and Gynae, Bangabandhu Sheikh Mujib Medical University, Dhaka.

b. Dr. Shiuly Chowdhury, Associate Professor, BSMMU, Dhaka.

c. Prof. Saleha Begum Chowdhury, Professor of Obst. and Gynae, BSMMU, Dhaka.

Address of Correspondence: Dr. Sharmeen Mahmood, Associate Professor, Department of Obst. and Gynae, Bangabandhu Sheikh Mujib Medical University, Dhaka.

Received: 5 July, 2015

Accepted: 7 June, 2016 presence of one or more of these indicators. The most common adverse perinatal outcome was birth asphyxia, low apgar score, stay at NICU, still birth and caesarean section for fetal distress. Of the 40 pregnancies in the study, 30 (75\%) showed abnormal C/U ratio. Among these, 32 (80\%) were SGA and 32 (80\%) had adverse perinatal outcome. Of the 30 out of 40 pregnancies that showed abnormal $\mathrm{C} / \mathrm{U}$ ratio $(<1.08)$, all 30 (80\%) were SGA and had adverse perinatal outcome. The results were correlated with parameters of fetal outcome. Inferences drawn from the study was that $\mathrm{C} / \mathrm{U}$ ratio is a tools for prediction of SGA fetuses and adverse perinatal outcome.

Keywords: Intrauterine growth retardation, pulsatility index, middle cerebral artery to umbilical artery pulsatility index ratio.

(J Bangladesh Coll Phys Surg 2016; 34: 145-150)

growth retardation fetus to allow for timely intervention is a main objective of antenatal care.

Umbilical artery and middle cerebral artery Doppler velocimetry is the most rigorous evaluation test among the noninvasive tests of fetal well being (Giles et al. 1985) ${ }^{4}$.

Placental insufficiency, whether primary or secondary to maternal factors such as hypertension, poor nutrition etc. is the most common cause of intrauterine growth retardation which is an important obstetric problem on account of the high association perinatal mortality and morbidity. It is essential to recognize placental insufficiency early so that its hazards can be reduced (Bano et al 2010) . $^{5}$

Doppler ultrasound allows a noninvasive assessment of fetal haemodynamics. Doppler ultrasound enable a better understanding of the haemodynamic changes and has therefore become one of the most important clinical tools for fetomaternal surveillance in high risk pregnancies.

Doppler investigation of umbilical arteries provides information concerning perfusion of the fetoplacental circulation. Doppler value is considered as normal when the cerebral-umbilical ratio is above 1.08 and below the value is considered abnormal. (Gramellini et al .1992) ${ }^{6}$. Bano et al. $2010^{5}$ Calculated the cerebral-umbilical ratio and found that it remains constant in the last 10 wks of 
pregnancy therefore it is used a single cut off value of 1.08 for all the cases of 30-41 wks of gestation.

The brain sparing effect is associated with an abnormal cerebral-umbilical ratio (<1.08). However, if hypoxia persists, the diastolic flow return to the normal level.

The cerebral- umbilical ratio remains constant during, the last 10 weeks of gestation and provide better diagnostic accuracy than either vessels pulsatility index alone (Fleischer et al, 1985) ${ }^{7}$. Doppler waveform abnormalities have been reported to be the most accurate predictor of poor neonatal outcome.

\section{Materials and Methods:}

This prospective study was carried out in department of Obstetrics and Gynaecology, Bangabandhu Sheikh Mujib Medical University (BSMMU), Dhaka, during the period of January 2014 to December 2014 to establish the usefulness of Color Doppler evaluation of $\mathrm{C} / \mathrm{U}$ pulsatility index ratio in diagnosis of IUGR and adverse perinatal outcome. Validity of the test were confirmed by determining sensitivity, specificity, accuracy, positive predictive value, negative predictive value.

Prior to commencement of this study the respective authority approved the research protocol. Proper permission was taken from the concerned department for this study. All the patients included in this study were informed about the nature of risk and benefit of the study.

Clinically diagnosed as a case of IUGR in BSMMU referred to the department of Radiology and Imaging of BSMMU were included.

Patients who are very sick with medical disorders like, hypertension, multiple pregnancies, patients refusing the procedure, difference in opinion of consultant radiologist regarding Doppler flow were excluded from the study.

For this purpose, a total number of 40 patients were enrolled in this study. Patients with suspected IUGR admitted in BSMMU referred to the department of Radiology and Imaging was included in this study. All the 40 patients were subjected to a repeated USG examination after 15 days of the initial study, when the finding of the initial study were reconfirmed. The 40 patients were subjected to color Doppler examination (Siemens Sonoline, G60s), using 3.5 MHZ transducer with $3 \mathrm{~mm}$ sample volume and medium filter. After technically satisfactory Doppler waveform had been recorded, the Pulsatility index of the umbilical artery (UA) and the middle cerebral artery (MCA) was noted and the ratio of the MCA and UA (the C/U ratio) were calculated. The pregnancies were followed up and the lineal perinatal outcome of each case were noted. Various intrapartum and neonatal indicators were used to assess the outcome, with an adverse outcome being defined as the presence of one or more of these indicators. All these information were collected in pre-designed structured data collection sheet.

\section{Sonographic variable}

\section{C/U pulsatility index Ratio= MCA PI/ UA PI.}

The cerebral umbilical $\mathrm{P} 1$ ratio remains constant in the last 10 weeks of the pregnancy therefore a single cutoff value of 1.08 considered normal. Below that value, velocimetry was considered abnormal, according to Gramellini et al 1985, Bano et al 2010.

\section{Diagnostic criteria:}

Normal C/U Pulsatility index ratio: $>1.08$

Abnormal C/U Pulsatility index ratio: $<1.08$

All the findings were analyzed by appropriate standard statistical method.

\section{Demographic and clinical variables:}

Gestational age at delivery.

Presence of IUGR (Birth weight $<10^{\text {th }}$ percentile) for gestational age

Normal Birth weight.

Normal outcome.

Apgar score $<7$ at 5 minutes.

Stay at NICU.

Still birth.

Birth asphyxia.

\section{Result:}

Out of 40 pregnancies in the study, who were evaluated by Doppler USG to identify the normal and abnormal cerebral umbilical pulsatility index ratio, most of them were found abnormal 30 (75 \%) and normal were $10(25 \%)$.

Out of 40 patients 32 had Small for gestational age and 08 had normal birth weight.

Out of 40 patients 32 had adverse perinatal outcome and 8 had normal outcome.

Among 30 cases, who had abnormal PI ratio, 28 cases were SGA and 2 had normal birth weight. Whereas, among normal pulsatility ratio only 1 was SGA. (Table-II)

Among 30 cases of abnormal PI ratio, all 30 had adverse perinatal outcome. Whereas among 10 cases of normal PI ratio only 2 had adverse perinatal outcome. (Table-III)

Tables II and__III depict the diagnostic performance of various Doppler flow indices in identifying SGA fetuses 


\section{Table-I}

Perinatal outcome and SGA (Small for gestational age) of the study population according to the cerebral-umbilical pulsatility index ratio (C/U PI ratio) $(n=40)$

\begin{tabular}{lccc} 
Serial Number & SGA & Normal Birth weight & Adverse perinatalOutcome present \\
\hline Normal PI ratio $(>1.08)(n=10)$ & 1 & 9 & $2(20 \%)$ \\
Abnormal PI ratio $(<1.08(n=30)$ & 28 & 2 & $30(80 \%)$ \\
\hline
\end{tabular}

\section{Table-II}

Diagnostic performance of pulsatility index and $\mathrm{C} / \mathrm{U}$ ratio for small for gestational age infants

\begin{tabular}{lccccccccc}
$\mathrm{n}=40$ & \multicolumn{3}{c}{ No. of findings } & & Sensitivity(\%) & Specificity(\%) & PPV(\%) & NPV(\%) & DA(\%) \\
& TP & FN & FP & TN & & & & \\
\hline C/U ratio & 28 & 1 & 2 & 9 & 96.55 & 81.81 & 93.33 & 90 & 92.5 \\
\hline
\end{tabular}

$\mathrm{PPV}=$ Positive predictive value, $\mathrm{NPV}=$ Negative predictive value, $\mathrm{DA}=$ Diagnositc accuracy, $\mathrm{TP}=\mathrm{True}$ positive, $\mathrm{FN}=\mathrm{False}$ negative, $\mathrm{FP}=$ False positive, $\mathrm{TN}=$ True negative.

\section{Table-III}

Diagnostic performance of pulsatility index and $\mathrm{C} / \mathrm{U}$ ratio for adverse perinatal outcome

\begin{tabular}{lcccccccrr}
$\mathrm{n}=40$ & \multicolumn{3}{c}{ No. of findings } & Sensitivity(\%) & Specificity(\%) & PPV(\%) & NPV(\%) & DA(\%) \\
& TP & FN & FP & TN & & & & \\
\hline C/U ratio & 30 & 2 & 0 & 8 & 96.7 & 77.7 & 93.7 & 87.5 & 92.5 \\
\hline
\end{tabular}

$\mathrm{PPV}=$ Positive predictive value, NPV = Negative predictive value, $\mathrm{DA}=$ Diagnositc accuracy, $\mathrm{TP}=$ True positive, FN= False negative, $\mathrm{FP}=$ False positive, $\mathrm{TN}=$ True negative .

and predicting adverse perinatal outcome. The sensitivity and positive predictive value (PPV) were higher for the $\mathrm{C} / \mathrm{U}$ ratio

\section{Discussion:}

IUGR is a pathological condition which is strongly related to the development and function of the uteroplacental and fetoplacental circulation. An adequate fetal circulation is necessary for fetal growth.

UA velocimetry correlates with hemodynamic changes in the fetoplacental circulation, with an increase in the number of the tertiary stem villi and arterial channels, as the fetoplacental compartment develop, the impedance in the UA decreases. A diastolic component in the UA flow velocity waveform appear during the early second trimester and progressively increase in the gestational age. A mature UA flow velocity waveform pattem shows low impedence and high diastolic flow with a low pulsatility index. During normal pregnancy, the MCA shows high resistance and low diastolic flow with an increase in the pulsatility index.

Gramellini et al. (1992) ${ }^{6}$ calculated the C/U ratio and found that remain constant in the last 10 weeks of pregnancy, therefore this study considered a single cutoff value of 1.08 for all cases of 30-41 weeks of gestation. Using the cut-off value, study population was divided into normal and abnormal.

In pregnancies with chronic fetal hypoxia, the blood volume in the fetal circulation is redistributed in favour of vitally important organs, the heart, kidneys, and brain. Vasodilatation of the MCA, with an increase diastolic flow through it, result in a decrease in its PI. The resulting hyperperfusion is considered pathological.

In IUGR, umbilical blood flow is significantly reduced, mainly due to changes in the placental vascular 


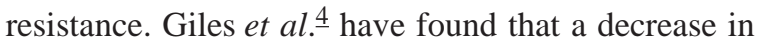
the number of resistance vessels in the tertiary stem villi in the placenta causes an increase in resistance, leading to decreased flow through the UA and an increase in the UA PI. This is described as umbilical placental insufficiency. Fleischer and Schulman ${ }^{7}$ have found that in IUGR complicated by pregnancy-induced hypertension, there is inadequate trophoblastic invasion of the spiral arteries, leading to increased resistance in the spiral arteries and decreased blood flow in the placental vascular bed and in the UA, thereby resulting in an increase in the UA PI. This is described as uteroplacental insufficiency. Several blood flow classes have been defined by Hofer et al. $\underline{8}$ to describe abnormal UA waveform patterns. Increasing pathological significance is ascribed to a decrease in diastolic flow, absence of diastolic flow and reversal of diastolic flow. All these patterns were associated with increased UA PI. Patients with absent end-diastolic volume (AEDV) and reverse end-diastolic volume (REDV) have the gravest outcome. Fetuses with AEDV require intensive surveillance as fetal well-being may deteriorate within a few days. Fetuses with REDV are most severely compromised. REDV is indicative of a preterminal fetal state.

In pregnancies with chronic fetal hypoxia, the blood volume in the fetal circulation is redistributed in favor of vitally important organs, i.e., the heart, kidneys and brain. Vasodilatation of the MCA, with an increase in diastolic flow through it, results in a decrease in its PI. The resulting hyperperfusion is considered pathological. This 'brain-sparing effect' is associated with an abnormal C/U ratio (<1.08). However, if hypoxia persists, the diastolic flow returns to the normal level. Presumably, this reflects a terminal decompensation in the setting of acidemia or brain edema. $\underline{8}$

This study dealing with a high-risk population, maximizing the sensitivity of a screening test is an ideal goal. For a screening system to be effective, it must be sensitive enough to detect the disease at an early stage so that treatment can bring a cure, sufficiently specific, acceptable to patients and adaptable to widespread screening, cost effective:

The Color Doppler study of C/U vessels has made an advancement in new generation sonography equipment and expertise, which has brought a revolution in the field of diagnostic imaging to diagnose IUGR prenatally. This non invasive imaging modality will be able to replace the other invasive diagnostic procedures.

This current study was carried out with an aim to establish the usefulness of $\mathrm{C} / \mathrm{U}$ Pulsatility index ratio in diagnosis of IUGR and prediction of adverse perinatal outcome.

Our results were more encouraging for the prediction of adverse perinatal outcome rather than diagnosing IUGR. It has been estimated that $41-86 \%$ of SGA babies can be detected with the routine use of symphysis-fundal height measurements. $\underline{-}$ According to one meta-analysis of USG fetal biometry, AC and estimated fetal weight (EFW) are the best predictors of fetal weight below the $10^{\text {th }}$ percentile. $\frac{10}{1}$ In high-risk populations, the sensitivity of using AC below the $10^{\text {th }}$ percentile is $73-95 \%$, whereas with EFW, the sensitivity is 43-89\%. In low-risk populations, the corresponding sensitivities are 48-64\% for AC and $31-73 \%$ for EFW. $\stackrel{11}{ }$ Biophysical profile is another method for detecting IUGR, with a sensitivity of $77.7 \%$. $\underline{12}$

We further found that when compared with other modalities, although Doppler velocimetry was relatively less sensitive for diagnosing SGA fetuses, because uteroplacental insufficiency is just one cause of IUGR, it proved to be very useful in predicting IUGR fetuses at risk for adverse perinatal morbidity and mortality. None of the other modalities has been as promising in detecting adverse perinatal outcome as Doppler velocimetry. Among the number of biophysical tests available to assess fetal well-being, the most common methods are amniotic fluid volume (AFV), biophysical profile scoring (BPS) and nonstress test (NST). A reduced AFV (either measured by maximum vertical pocket $<2 \mathrm{~cm}$ or a four-quadrant amniotic fluid index (AFI) $<5$ cm poorly correlates with the actual AFV and does not accurately predict adverse perinatal outcome. A review of 18 studies indicated that an AFI $<5 \mathrm{~cm}$ was associated with an increased risk of Cesarean section for fetal distress (relative risk [RR] 2.2; 95\% confidence interval [CI] 1.5-3.4) and Apgar score of $<7$ at $5 \mathrm{~min}$ (RR 5.2; 95\% CI 2.4-11.3), but not with neonatal acidosis. 13 Large observational studies have shown an association between reduced AFV and perinatal morbidity and mortality, but the predictive value is poor $(<10 \%)$ and, also, there is little evidence to support intervention with 
isolated oligohydramnios (with a normal UA Doppler)..$^{14}$ Yoon et al. $\underline{15}$ attempted to compare the performance of BPS and UA Doppler velocimetry in the identification of fetal acidemia, hypoxemia and hypercarbia as determined by $\mathrm{PH}$ and gas analysis of fetal blood obtained by cordocentesis in 24 patients. Although they found a strong relationship between the degree of fetal acedemia and hypercarbia and the results of UA Doppler velocimetry and BPS, Doppler velocimetry proved to be a better explanatory variable for these outcomes than the BPS. $\frac{15}{}$ Another study was performed by Gonzalez et al. $\underline{16}$ to compare the efficacy of NST, BPS and abnormal Doppler findings in predicting adverse perinatal outcomes in IUGR. The PPVs of abnormal Doppler for respiratory distress syndrome and the composite of adverse outcomes were $36 \%$ and $42 \%$, respectively. Of the testing modalities compared, only abnormal Doppler significantly predicted respiratory distress syndrome and the composite of adverse outcome. Hence, they concluded that in cases of IUGR, the presence of abnormal Doppler was the best predictor of adverse perinatal outcome. $\frac{16}{}$ Padmagirison Radhika et al. $\underline{17}^{17}$ conducted prospective antenatal fetal surveillance in 55 women to compare the efficacy of Doppler velocimetry and NST in predicting fetal compromise in utero in cases of severe pre-eclampsia or IUGR. There were 29 cases with abnormal Doppler and 20 cases with abnormal NST. In addition, Doppler abnormalities preceded NST changes with a lead time of 4.14 days and there were 10 perinatal deaths, six of which occurred in the group where both the tests were abnormal. They concluded that Doppler identifies fetal compromise earlier than NST. The lead time helps to plan delivery in preterm compromised pregnancies, resulting in better perinatal survival. $\underline{\underline{17}}$

Validity test was done be calculating sensitivity, specificity, accuracy, positive predictive value (PPV) and negative predictive value (NPV) respectively. The present study findings were discussed and compared with previously published relevant studies.

Gramellini et $a 1^{6}$. (1992) had shown their study by a C/ $\mathrm{U}$ pulsatility index ratio of less than 1.08 had a sensitivity of $68 \%$, specificity $98.4 \%$. Positive predictive value $94.4 \%$, Negative predictive value $88.8 \%$ and Diagnostic accuracy $90 \%$ for predicting adverse perinatal outcomes in IUGR.
Schulman et $a 1^{7}$. (1999) reported the sensitivity of 63\% for predicting adverse perinatal outcomes in IUGR.

Obido et $a 1^{16}$. (2005) reported by a C/U Pl 'ratio of less than 1.08 had a sensitivity of $90 \%$ predicting adverse perinatal outcomes in IUGR.

Bano et $a 1^{5}$. (2010) Studied by a C/U P1 ratio of less than 1.08 had a sensitivity of $83.3 \%$, specificity $100 \%$, Positive predictive value $100 \%$. Negative predictive value $94.3 \%$ and Diagnostic accuracy $95.6 \%$ for predicting adverse perinatal outcomes in IUGR.

In this study by using a $\mathrm{C} / \mathrm{U}$ pulsatility index ratio of less than 1.08 and sensitivity is $96.7 \%$, specificity $77.7 \%$, Positive predictive value $93.7 \%$, Negative predictive value $87.5 \%$ and Diagnostic accuracy $92.5 \%$ for predicting adverse adverse perinatal outcomes in IUGR.

In asymmetrical IUGR there is high umbilical artery PI and low middle cerebral artery PI. As a result, the $\mathrm{C} / \mathrm{U}$ ratio is lower than normal growth retarded fetuses.

Bano et $a l^{5}$. (2010) and Obido et $a l^{16}$ (1992), have observed in their series that Doppler was significantly correlated with adverse perinatal outcome where sensitivity ranged from $83-90 \%$, specificity from $71-100 \%$. Thus the parameters provide strong evidence that Doppler analysis is of great value in evaluation of prenatal diagnosis of fetal at risk in IUGR.

The cerebral umbilical Pl ratio incorporates data of both placental status (umbilical artery) and fetal response (Middle cerebral artery) in the prediction of adverse outcomes.

In this study, comparing the use of the $\mathrm{C} / \mathrm{U}$ pulsatility index ratio, we found similar efficiency in the prediction of IUGR and adverse perinatal outcomes. This implies that perinatal centers can use this method in the evaluation of cases of IUGR. According to cerebral umbilical Pl value 30 (75\%) were diagnosed as abnormal and 10 (25\%) as normal flow pattern.

Perinatal findings showed 32 were SGA and 8 have normal birth weight.

The validity of cerebral umbilical Pl ratio for diagnosis for diagnosis of IUGR were studied by calculating sensitivity, specificity, accuracy PPV, NPV which were 96.55\%, 81.81\%, 92.5\%, 93.33\% and 90\% respectively.

The validity of cerebral umbilical Pl ratio for adverse perinatal outcome were studied by calculating 
sensitivity, specificity, accuracy, PPV, NPV which were 96.7\% 77.7\%, 92.5\%, 93.7\% and 87.5\% respectively.

Color Doppler study over other imaging modalities is preferred due to its relative accuracy, non invasiveness, lack of ionizing radiation, less expensiveness and availability.

\section{Conclusion:}

In this study Color Doppler findings of cerebral-umbilical vessels and the validity test are almost identical as observed by other investigators compared with perinatal findings, so it can be concluded that color Doppler evaluation of MCA and UA Pl ratio is an useful modality in diagnosis of IUGR and prediction of adverse perinatal outcome.

\section{References:}

1. Wolfe, HM \& Gross, TL 1989, Increase risk to the growth retardated fetus, Intrauterine growth retardation. Chocage, Plll.

2. Berkowitz, GS, Mehalek, KE, Chitkara, U, Rosenberg, .1, Cogswell, C \& Berkowitz RL 1988, Doppler umbilical velocimetry $1 \mathrm{n}$ the prediction of adverse outcome in pregnancies Risk for inrauterine growth retardation, Obster Gynecol, vol. 71,p. 742.

3. Kok, JH, Denouden, AL, Verloove-Vanhoriek, SP \& Brand R 1998, Outcome of very preterm small for gestational age infants: the first none years of life, $\mathrm{Br} \mathrm{J}$ Obstet Gynecol, vo1.- 10,pp. 162-168.

4. Giles WB, Trudinger BJ, Baird PJ. Fetal umbilical flow velocity waveform and placental resistance pathological correlation. Br J Obstet Gynaecol. 1985;92:31-8.

5. Bano's, ChaudharyN, Pande,s, IVlehta,VL, \& Sharma,AK 2010, Indian journal of radiology and Imaging, vol. 20, pp. 20-25.

6. Gramellini,D, Folli,MC, Raboni,S, Vadora,E \& Merialdi,A 1992, Cerebral umbilical Doppler rate as a predictor of adverse perinatal outcome, Obstet Gynecol, vol. 79,pp. 416-20.
7. Fleischer,A, schulman,H, Farmakides, G, Bracero,L, Blattner, P \& Randolph, G 1985, Umbilical artery flow velocity waveforms and intrauterine growth retardation, Am J Obstet gynecol, vol. 151, pp. 502-505.

8. Tatjana Reihs, Matthias Hofer. In: Obstetrics and gynecology Sonography. 2nd ed. Matthias Hofer., editor. New York: Thieme; 2004. pp. 61-72.

9. Quaranta P, Currell R, Redman CW, Robinson JS. Prediction of small-for-dates infants by measurement of symphysis-fundal height. $\mathrm{Br} \quad \mathrm{J}$ Obstet Gynaecol. 1981;88:115-9.

10. Chang TC, Robson SC, Boys RJ, Spencer JA. Prediction of the small for gestational age infant: Which ultrasonic measurement is best? Obstet Gynecol. 1992;80:1030-8.

11. Warsof SL, Cooper DJ, Little D, Campbell S. Routine ultrasound screening for antenatal detection of intrauterine growth retardation. Obstet Gynecol. 1986;67:33-9.

12. Afzal A, Nasreen K. Biophysical score in high risk pregnancy. Prof Med J. 2006;13:362-9.

13. Chauhan SP, Sanderson M, Hendrix NW, Magann EF, Devoe LD. Perinatal outcome and amniotic fluid index in the antepartum and intrapartum periods: A metaanalysis. Am J Obstet Gynecol. 1999;181:1473-8.

14. Forfar J, Mclntosh N, Arneil GC, et al. Forfar and Arneil text book of pediatrics. Google book result.2003:167-8.

15. Yoon BH, Romero R, Roh CR, Kim SH, Ager JW, Syn HC, et al. Relationship between the fetal biophysical profile score, umbilical artery Doppler velocimetry, and fetal blood acid-base status determined by cordocentesis. Am J Obstet Gynecol. 1993;169:1586-94.

16. Gonzalez JM, Stamilio DM, Ural S, Macones GA, Odibo AO. Relationship between abnormal fetal testing and adverse perinatal outcomes in intrauterine growth restriction. Am J Obstet Gynecol.2007;196:48-51.

17. Padmagirison R, Rai L. Fetal doppler versus NST as predictors of adverse perinatal outcome in severe preeclampsia and fetal growth restriction. J Obstet Gynecol. 2006;56:134-8. 